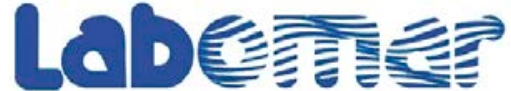

Arquivos de Ciências do Mar

\section{CARACTERIZAĢÃO DA FAUNA VÁGIL ASSOCIADA AO CULTIVO SUSPENSO DE MEXILHÕES}

\author{
Characterization of vagile fauna associated \\ with suspended mussel farms \\ Fausto Silvestri ${ }^{1,2 *}$, Ligia Coletti Bernadochi ${ }^{1}$, Carina Waiteman Rodrigues ${ }^{2}$, \\ Mauricio Shimabukuro ${ }^{2}$, Paulo Márcio Santos Costa ${ }^{1}$, Alexander Turra ${ }^{2}$ \\ ${ }^{1}$ FIPERJ - Fundação Instituto de Pesca do Estado do Rio de Janeiro, Escritório Regional da Costa Verde, \\ Rua do Comércio ${ }^{\circ}{ }^{10}$, Centro, CEP 23970-000, Angra dos Reis (RJ), Brasil. \\ ${ }^{2}$ Instituto Oceanográfico, Universidade de São Paulo, São Paulo (SP), Brasil. \\ *Autor para correspondência silvestrifausto@hotmail.com
}

\begin{abstract}
RESUMO
Fazendas de mexilhões oferecem abrigo e alimentação para uma grande variedade de organismos tornando-se um ambiente favorável para o estabelecimento de populações biológicas. Todavia a presença destes organismos no cultivo gera consequências ecológicas e zootécnicas, positivas ou negativas. Nesse estudo foi realizada a caracterização da fauna vágil associada a uma área de produção artesanal de mexilhões Perna perna (L. 1758) no sudeste do Brasil, representando uma importante iniciativa para que se possa estudar os efeitos da presença destes organismos tanto para os cultivos como para o ambiente ao qual eles estão inseridos. Ao longo de um ano foram identificados 41.082 indivíduos vágeis, totalizando 109 diferentes táxons diretamente associados às cordas de mexilhões. A estrutura da comunidade vágil variou ao longo do período de estudo indicando a dominância de anfípodes em todos os períodos analisados. Os resultados deste estudo indicaram que o cultivo suspenso de mexilhões praticado em uma área costeira desempenhou uma função estratégica na atração e no refúgio de espécies vágeis.
\end{abstract}

Palavras-chave: aquicultura, mitilicultura, Perna perna, biodiversidade, fouling.

\begin{abstract}
Mussel farms provide shelter and food for a wide variety of organisms making a favorable environment for the establishment of biological populations. However, the presence of these
\end{abstract}

Recebido em: 25/05/2018

Aprovado em: 13/09/2018

Publicado online em: 25/03/2019 
organisms in mussel farms generates ecological and zootechnical consequences, both positive and negative. In the present study, we performed the first characterization of the vagile fauna associated to small brown mussels Perna perna (L.1758) farms in southeastern Brazil. This represents an important contribution to understanding the effects of the presence of these organisms both in the mussel farms and in the environment that they are inserted. During one year, 41.082 organisms were identified, from a total of 109 different taxa that were directly associated with the strings of mussels. The community structure of the vagile varied throughout the study, indicating the dominance of amphipods in all periods analyzed. Therefore, results indicated that the suspended mussel culture performed in a coastal area played a strategic role to the attraction and refuge of vagile species.

Key words: aquaculture, mussel culture, Perna perna, biodiversity, fouling.

\section{INTRODUÇÃO}

Estudos ecológicos envolvendo a maricultura podem fornecer subsídios para que os cultivos possam se tornar cada vez mais integrados ao meio onde estão inseridos. Quando localizados em áreas com baixo hidrodinamismo e em condição de imersão constante, os cultivos suspensos de moluscos bivalves proporcionam a formação de micro hábitats que fornecem condições adequadas para a colonização de muitas espécies epibiontes, além de oferecerem refúgio e alimento para uma série de organismos a eles associados (Gibbs, 2004; Tallman \& Forrester, 2007; Sá et al., 2007; Watson et al., 2009; Freitas \& Velastin, 2010; Lacoste et al., 2014).

Determinados estudos indicam que áreas onde são praticados cultivos de mexilhões são propicias para a prática da pesca, tendo em vista a maior concentração de peixes que ocorre nestes locais (Souza-Conceição et al., 2003; Morrisey et al., 2006; Clynick et al., 2008; Bot-Neto \& Rosa, 2017). Além disso, a biodeposição produzida pelos cultivos de moluscos permite o suporte de uma densa epifauna na área sob influencia das fazendas marinhas, atraindo consequentemente, uma fauna demersal associada (Callier et al., 2009; AlonsoPérez et al., 2010; Silvestri \& Turra, 2013).

Na rotina de manejo da mitilicultura, após a despesca retira-se e descarta-se toda a fauna associada aos mexilhões, composta por organismos incrustantes e aqueles que vagam pela superfície das cordas mexilhoneiras. Tais organismos podem afetar a produtividade dos mexilhões pela competição por espaço e alimento (Rajbanshi \& Pederson, 2007; Comeau et al., 2015), predação (Kamermans et al., 2009; Peteiro et al., 2010), obstrução das valvas (Witman \& Suchanek, 1984), podendo comprometer a aparência e aumentar os custos de produção (Fitridge et al., 2012) e também contribuir para o incremento da quantidade de detritos no sedimento por biodeposição (McKindsey et al., 2009). Com relação ao mexilhão Perna perna, principal espécie de molusco cultivada no Brasil, a fauna associada pode representar até $30 \%$ do peso total da corda mexilhoneira (Marenzi \& Branco, 2006).

No Brasil existem poucas informações sobre a fauna associada ao cultivo de mexilhões, focadas principalmente em organismos epibiontes (Metri et al., 2002; Sá et al., 2007) e espécies invasoras (Rocha et al., 2002). Considerando que o cultivo de mexilhões pode atuar como um importante atrator da fauna vágil, podendo incrementar a biodiversidade em áreas onde estão inseridas as fazendas marinhas, este estudo visa caracterizar a fauna vágil 
associada ao cultivo suspenso de mexilhões em uma área costeira no litoral norte do Estado de São Paulo, bem como discutir sobre as implicações ecológicas e suas relações com a produção de mexilhões.

\section{MATERIAL E MÉTODOS}

Este estudo foi conduzido na Praia da Cocanha, situada no município de Caraguatatuba, litoral norte do estado de São Paulo (Figura 1). O local selecionado para este estudo é o principal polo produtor de mexilhões do Estado de São Paulo, onde o cultivo é realizado em pequenas fazendas marinhas com até $2.000 \mathrm{~m}^{2}$ de área total. A produção de moluscos é realizada de modo artesanal, por caiçaras, organizados por meio de uma associação de maricultores locais (Silvestri et al., 2008).

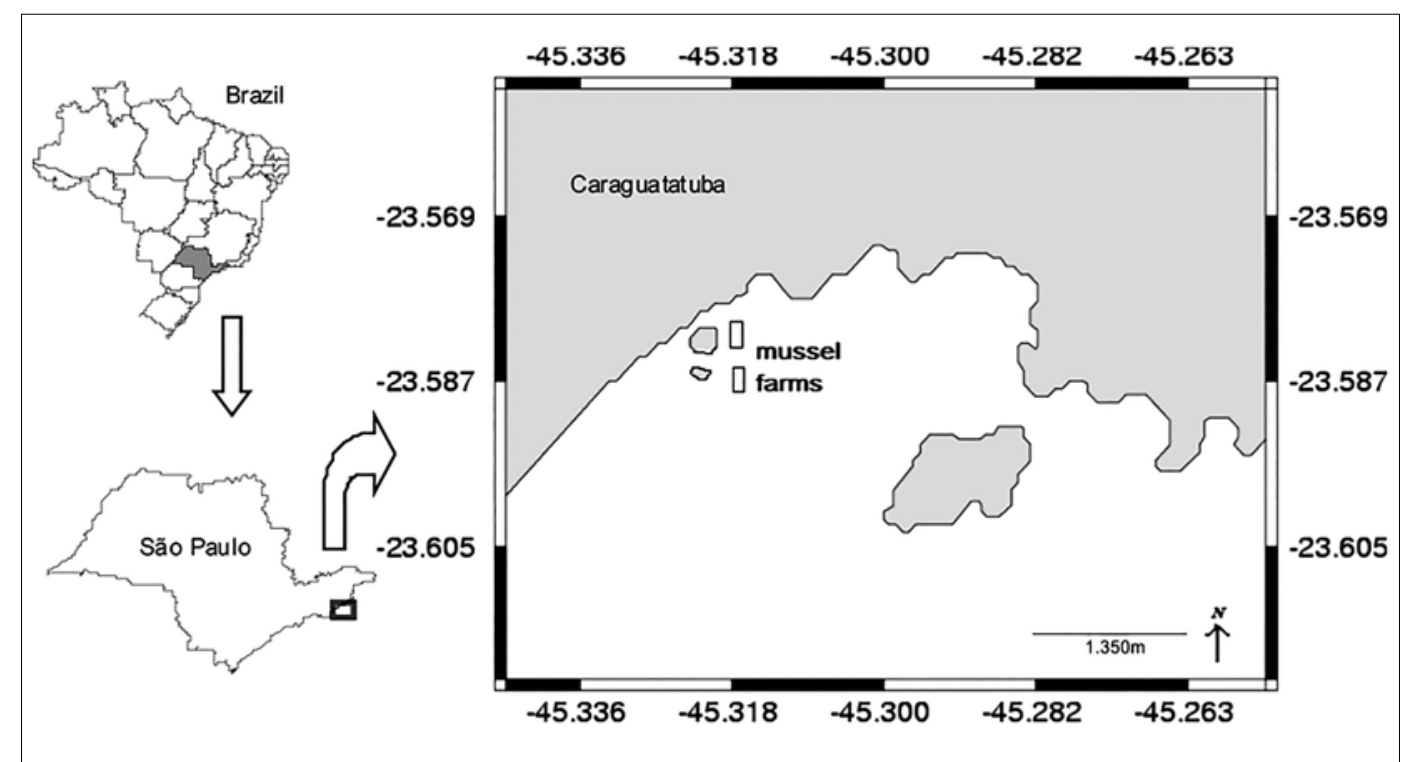

Figura 1 - Localização geral da área de estudo.

Desde 1988, no local são cultivados mexilhões Perna perna em espinhéis posicionados paralelamente na superfície da água, suspensos por flutuadores plásticos e ancorados nas extremidades por poitas de concreto. Fixadas aos espinhéis, as cordas mexilhoneiras apresentam cerca de dois metros de comprimento linear, com espaçamento de meio metro entre as cordas. Via de regra, as cordas recém-semeadas permanecem submersas por um período de cerca de oito meses quando os mexilhões atingem tamanho comercial com aproximadamente sete centímetros de comprimento (Marques, 1998).

A fim de caracterizar a fauna vágil associada ao cultivo suspenso de mexilhões foram realizadas ao longo de um ano quatro amostragens trimestrais nos meses de maio, agosto, novembro e fevereiro.

Em cada amostragem nove cordas mexilhoneiras foram aleatoriamente selecionadas por meio de sorteio e retiradas da água através de mergulho livre com o auxilio de bolsas coletoras especialmente desenvolvidas para evitar a fuga de organismos vágeis. Em seguida, as bolsas contendo as cordas mexilhoneiras foram colocadas em uma mesa de inox para realizar a limpeza e a triagem do material biológico. Foram selecionados somente os 
organismos vágeis e sedentários sendo descartada toda a fauna séssil. As amostras foram fixadas em formalina 10\% e posteriormente conservadas em álcool 70\%. Uma segunda triagem foi realizada em laboratório classificando os organismos em grandes grupos sendo em seguida encaminhados para identificação ao menor nível taxonômico possível.

A fim de caracterizar a comunidade de organismos vágeis presentes nas cordas mexilhoneiras avaliou-se inicialmente a abundância relativa dos principais grupos taxonômicos, considerando o somatório de indivíduos descritos em todas as nove cordas mexilhoneiras para cada período de amostragem. Complementarmente, para cada um dos períodos, foram determinadas as médias aritméticas e o desvio padrão $(n=9)$ dos seguintes descritores de comunidade: abundância $(\mathrm{N})$, representado pelo número total de indivíduos encontrados em cada corda mexilhoneira; riqueza (S), referente ao número total de espécies identificadas; Índice de Diversidade de Shannon $\left(\mathrm{H}^{\prime}\right), H^{\prime}=-\Sigma p_{i} \ln \left(p_{i}\right)$, onde $\mathrm{p}_{\mathrm{i}}$ é a proporção de indivíduos da espécie i; Índice de dominância de Simpson (D), onde $D=\Sigma p_{i}^{2}$ e expresso na forma de $1 / \mathrm{D}$; e Equabilidade de Pilou $\left(\mathrm{J}^{\prime}\right)$, onde $J^{\prime}=H / \ln (S)$.

As diferenças entre os descritores foram comparadas entre períodos de coleta $(n=4)$ por meio de testes não paramétricos de Kruskall-Wallis, seguidas do teste SNK para comparações múltiplas a posteriori (Underwood, 1997).

\section{RESULTADOS}

No total foram identificados 41.082 indivíduos, representando 109 diferentes táxons. A fauna vágil associada às cordas mexilhoneiras foi caracterizada pela dominância de anfípodes em todos os períodos analisados. Outros táxons representativos foram Polychaeta, Decapoda, Pisces e Mollusca (Figura 2).

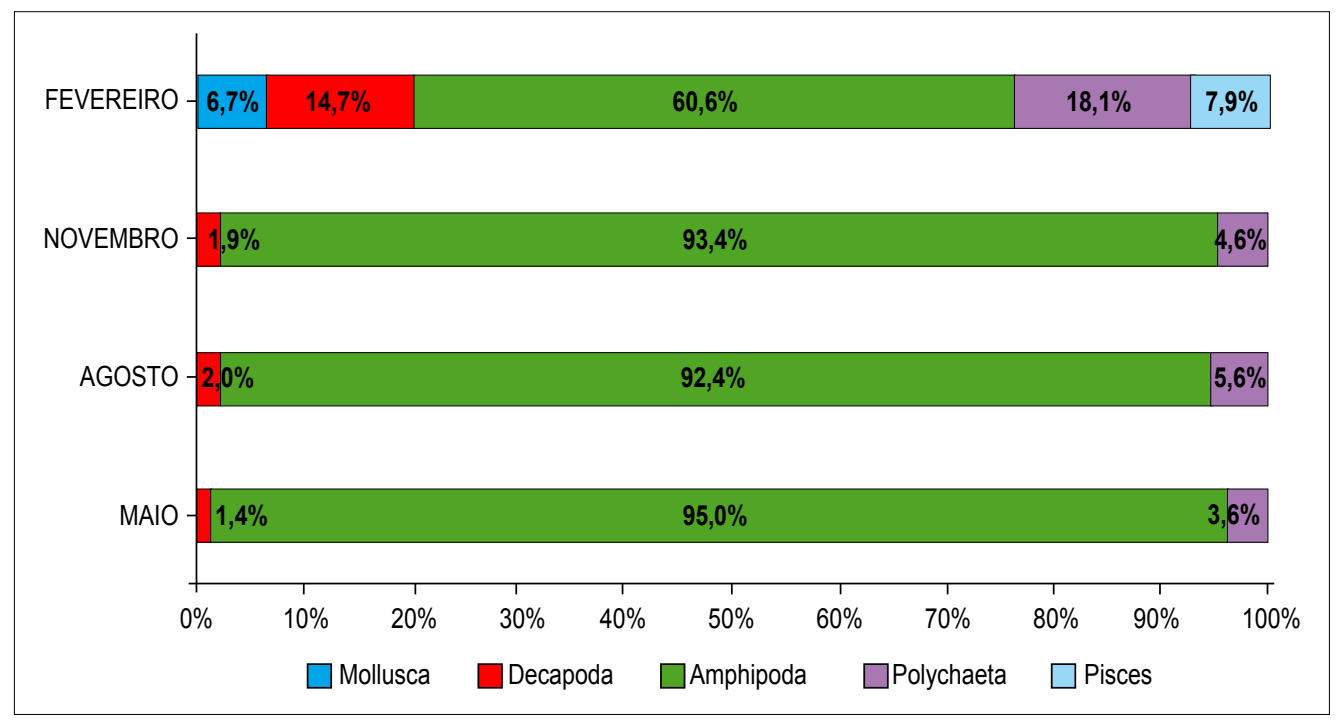

Figura 2 - Abundância relativa dos principais grupos taxonômicos de fauna vágil associados às cordas mexilhoneiras na Praia da Cocanha.

Podocerus brasiliensis (18,88\%) Caprella equilibra (15,63\%), Dulichiella anisochir (13,23\%) e Jassa marmorata $(10,52 \%)$ foram às espécies mais representativas ao longo do estudo. Pseudonereis palpata (5,06\%) e Leodice rubra (0,61\%), Hypleurochilus fissicornis (0,97\%), 
Pachycheles laevidactilus (0,54\%) e Acantholobulus schmitti (0,49\%) foram os táxons mais representativos entre seus respectivos grupos (Tabela 1).

De maneira geral, 28 espécies ocorreram em todos os períodos amostrais, sendo 01 molusco (Gastropoda), 23 artrópodes (12 Decapoda, 10 Amphipoda e 01 Tanaidacea), 03 anelídeos (Polychaeta) e 01 cordado (Actynopiterygii - Pisces).

Tabela 1 - Fauna vágil associada a cordas mexilhoneiras na Praia da Cocanha: abundancia numérica nos meses de amostragem, abundancia total (n) e relativa (\%).

\begin{tabular}{|c|c|c|c|c|c|c|c|}
\hline \multirow{2}{*}{$\frac{\text { Filo/Classe }}{\text { MOLLUSCA }}$} & \multirow[t]{2}{*}{ Táxon } & \multirow[t]{2}{*}{ maio } & \multirow[t]{2}{*}{ agosto } & \multicolumn{2}{|c|}{ novembro fevereiro } & \multirow[t]{2}{*}{$\mathbf{N}$} & \multirow[t]{2}{*}{$\%$} \\
\hline & & & & & & & \\
\hline \multirow[t]{8}{*}{ BIVALVIA } & Hiatella arctica (Linnaeus, 1767) & 0 & 0 & 0 & 8 & 8 & $0,02 \%$ \\
\hline & Sphenia fragilis (H. Adams \& A. Adams, 1854) & 0 & 0 & 2 & 6 & 8 & $0,02 \%$ \\
\hline & Arcopsis adamsi (Dall, 1886) & 1 & 0 & 0 & 0 & 1 & $0,00 \%$ \\
\hline & Barbatia candida (Helbling, 1779) & 0 & 1 & 0 & 0 & 1 & $0,00 \%$ \\
\hline & Lunarca ovalis (Bruguière, 1789) & 0 & 0 & 0 & 6 & 6 & $0,01 \%$ \\
\hline & Musculus lateralis (Say, 1822) & 0 & 0 & 0 & 1 & 1 & $0,00 \%$ \\
\hline & Paraleptopecten bavayi (Dautzemberg, 1900) & 0 & 0 & 2 & 5 & 7 & $0,02 \%$ \\
\hline & Pteria colymbus (Röding, 1798) & 0 & 0 & 1 & 0 & 1 & $0,00 \%$ \\
\hline \multirow[t]{24}{*}{ GASTROPODA } & Tegula viridula (Orbigny, 1840) & 0 & 0 & 0 & 3 & 3 & $0,01 \%$ \\
\hline & Eulithidium affine (C.B. Adams, 1850) & 0 & 0 & 0 & 3 & 3 & $0,01 \%$ \\
\hline & Lucapinella limatula (Reeve, 1850) & 2 & 6 & 22 & 92 & 122 & $0,30 \%$ \\
\hline & Lucapina sowerbii (Sowerby, 1835) & 0 & 0 & 0 & 2 & 2 & $0,00 \%$ \\
\hline & Fissurella rosea (Gmelin, 1791) & 0 & 0 & 0 & 16 & 16 & $0,04 \%$ \\
\hline & Fissurella clenchi Farfante, 1943 & 0 & 0 & 3 & 7 & 10 & $0,02 \%$ \\
\hline & Diodora cayenensis (Lamarck, 1822) & 0 & 0 & 0 & 5 & 5 & $0,01 \%$ \\
\hline & Cerithiopsis iota (C.B. Adams, 1845) & 0 & 0 & 0 & 67 & 67 & $0,16 \%$ \\
\hline & Epitonium cf. pseudonanum (Bouchet \& Waren, 1986) & 0 & 0 & 0 & 1 & 1 & $0,00 \%$ \\
\hline & Bittiolum varium (Pfeiffer, 1840) & 0 & 0 & 0 & 4 & 4 & $0,01 \%$ \\
\hline & Anachis aff. sparsa (Revee, 1859) & 0 & 0 & 0 & 6 & 6 & $0,01 \%$ \\
\hline & Anachis sertulariarum Orbigny, 1841 & 0 & 0 & 0 & 5 & 5 & $0,01 \%$ \\
\hline & Anachis isabellei (Orbigny, 1841) & 0 & 1 & 0 & 4 & 5 & $0,01 \%$ \\
\hline & Mitrella dichroa (G.B. Sowerby, 1844) & 0 & 0 & 0 & 1 & 1 & $0,00 \%$ \\
\hline & Olivella minuta (Link, 1807) & 0 & 0 & 0 & 1 & 1 & $0,00 \%$ \\
\hline & Stramonita brasiliensis Claremont \& B. G. Reid 2011 & 0 & 1 & 1 & 15 & 17 & $0,04 \%$ \\
\hline & Pisania sp. & 0 & 0 & 0 & 1 & 1 & $0,00 \%$ \\
\hline & Cymatium parthenopeum (Von Salis, 1793) & 0 & 0 & 1 & 2 & 3 & $0,01 \%$ \\
\hline & Fargoa bushiana (Bartsch, 1909) & 0 & 0 & 0 & 5 & 5 & $0,01 \%$ \\
\hline & Iselica globosa (H. C. Lea, 1843) & 0 & 0 & 0 & 8 & 8 & $0,02 \%$ \\
\hline & Bulla sp. & 0 & 0 & 0 & 1 & 1 & $0,00 \%$ \\
\hline & Siphonaria hispida Hubendick, 1946 & 0 & 0 & 0 & 1 & 1 & $0,00 \%$ \\
\hline & Doris verrucosa Linnaeus, 1758 & 2 & 0 & 0 & 0 & 2 & $0,00 \%$ \\
\hline & Babakina festiva (Roller, 1972) & 0 & 0 & 0 & 2 & 2 & $0,00 \%$ \\
\hline POLYPLACOPHORA & Ischnochiton striolatus (Gray, 1828) & 0 & 0 & 0 & 2 & 2 & $0,00 \%$ \\
\hline \multicolumn{8}{|l|}{ ARTHROPODA } \\
\hline \multirow[t]{19}{*}{ MALACOSTRACA } & Alpheus sp. & 0 & 0 & 0 & 1 & 1 & $0,00 \%$ \\
\hline & Alpheus formosus Gibbes, 1850 & 0 & 0 & 0 & 2 & 2 & $0,00 \%$ \\
\hline & Synalpheus fritzmuelleri Coutière, 1909 & 0 & 0 & 0 & 14 & 14 & $0,03 \%$ \\
\hline & Synalpheus townsendi Coutière, 1909 & 0 & 0 & 0 & 5 & 5 & $0,01 \%$ \\
\hline & Periclimenes sp. & 0 & 0 & 0 & 20 & 20 & $0,05 \%$ \\
\hline & Pontonia sp. & 0 & 0 & 0 & 17 & 17 & $0,04 \%$ \\
\hline & Pachycheles laevidactilus Ortmann, 1892 & 0 & 2 & 98 & 123 & 223 & $0,54 \%$ \\
\hline & Pachycheles monilifer (Dana, 1852) & 0 & 0 & 5 & 12 & 17 & $0,04 \%$ \\
\hline & Porcellana sayana (Leach, 1820) & 2 & 0 & 8 & 45 & 55 & $0,13 \%$ \\
\hline & Petrolisthes armatus (Gibbes, 1850) & 0 & 0 & 1 & 1 & 2 & $0,00 \%$ \\
\hline & Petrolisthes galathinus (Bosc, 1802) & 0 & 0 & 0 & 1 & 1 & $0,00 \%$ \\
\hline & Pisidia brasiliensis Haig in Rodrigues da Costa, 1968 & 1 & 4 & 4 & 19 & 28 & $0,07 \%$ \\
\hline & Cronius ruber (Lamarck, 1818) & 3 & 0 & 0 & 2 & 5 & $0,01 \%$ \\
\hline & Achelous rufiremus (Holthuis, 1959) & 2 & 0 & 0 & 0 & 2 & $0,00 \%$ \\
\hline & Callinectes sp. & 0 & 0 & 0 & 1 & 1 & $0,00 \%$ \\
\hline & Charybdis hellerii A. Milne Edwards, 1867 & 3 & 1 & 41 & 50 & 95 & $0,23 \%$ \\
\hline & Eurypanopeus abbreviatus (Stimpson, 1860) & 1 & 0 & 0 & 0 & 1 & $0,00 \%$ \\
\hline & Hexapanopeus sp. & 0 & 0 & 0 & 28 & 28 & $0,07 \%$ \\
\hline & Hexapanopeus paulensis Rathbun, 1930 & 11 & 50 & 27 & 48 & 136 & $0,33 \%$ \\
\hline
\end{tabular}


(continuação Tabela 1)

\begin{tabular}{|c|c|c|c|c|c|c|c|}
\hline & Acantholobulus schmitti (Rathbun, 1930) & 43 & 24 & 76 & 58 & 201 & $0,49 \%$ \\
\hline & Menippe nodifrons Stimpson, 1859 & 11 & 8 & 22 & 61 & 102 & $0,25 \%$ \\
\hline & Panopeus americanus Saussure, 1857 & 13 & 36 & 50 & 24 & 123 & $0,30 \%$ \\
\hline & Panopeus austrobesus Williams, 1983 & 1 & 2 & 4 & 3 & 10 & $0,02 \%$ \\
\hline & Panopeus sp. & 1 & 0 & 2 & 43 & 46 & $0,11 \%$ \\
\hline & Panopeus occidentalis Saussure, 1857 & 1 & 3 & 4 & 8 & 16 & $0,04 \%$ \\
\hline & Acantholobulus bermudensis (Benedict \& Rathbun, 1891 & 0 & 0 & 0 & 3 & 3 & $0,01 \%$ \\
\hline & Pilumnus dasypodus Kingsley, 1879 & 6 & 8 & 7 & 3 & 24 & $0,06 \%$ \\
\hline & Pilumnus diomedeae Rathbun, 1894 & 1 & 0 & 1 & 0 & 2 & $0,00 \%$ \\
\hline & Pilumnus reticulatus Stimpson, 1860 & 4 & 3 & 3 & 9 & 19 & $0,05 \%$ \\
\hline & Pilumnussp. & 0 & 0 & 3 & 0 & 3 & $0,01 \%$ \\
\hline & Pilumnus spinosissimus (Rathbun, 1898) & 1 & 26 & 6 & 10 & 43 & $0,10 \%$ \\
\hline & Pilumnoides hassleri A. Milne Edwards, 1880 & 1 & 0 & 1 & 0 & 2 & $0,00 \%$ \\
\hline & Microphrys bicornutus (Latreille, 1825) & 0 & 1 & 0 & 0 & 1 & $0,00 \%$ \\
\hline & Pachygrapsus transversus (Gibbes, 1850) & 1 & 1 & 10 & 7 & 19 & $0,05 \%$ \\
\hline & Cirolana sp. & 1 & 1 & 2 & 0 & 4 & $0,01 \%$ \\
\hline & Amphithoe ramondi Audouin, 1826 & 30 & 170 & 1550 & 433 & 2183 & $5,31 \%$ \\
\hline & Monocorophium acherusicum Costa, 1851 & 1208 & 691 & 619 & 6 & 2524 & $6,14 \%$ \\
\hline & Podocerus brasiliensis (Dana, 1853) & 1709 & 1033 & 4523 & 490 & 7755 & $18,88 \%$ \\
\hline & Jassa marmorata Holmes, 1903 & 68 & 1147 & 3068 & 38 & 4321 & $10,52 \%$ \\
\hline & Ericthonius punctatus (Bate, 1857) & 1378 & 287 & 834 & 284 & 2783 & $6,77 \%$ \\
\hline & Photis longicaudata (Bate \& Westwood, 1862) & 253 & 175 & 252 & 206 & 886 & $2,16 \%$ \\
\hline & Caprella equilibra Say, 1818 & 869 & 2453 & 3099 & 0 & 6421 & $15,63 \%$ \\
\hline & Caprella dilatata Krøyer, 1843 & 41 & 16 & 236 & 0 & 293 & $0,71 \%$ \\
\hline & Elasmopus pectenicrus (Bate, 1862) & 468 & 523 & 809 & 358 & 2158 & $5,25 \%$ \\
\hline & Dulichiella anisochir (Krøyer, 1845) & 111 & 1399 & 3365 & 562 & 5437 & $13,23 \%$ \\
\hline & Leucothoe sp. & 15 & 8 & 36 & 41 & 100 & $0,24 \%$ \\
\hline & Stenothoe valida Dana, 1852 & 1374 & 100 & 89 & 130 & 1693 & $4,12 \%$ \\
\hline & Lysianassidae Gen. sp. & 1 & 0 & 0 & 0 & 1 & $0,00 \%$ \\
\hline & Hyale niger (Haswell, 1879) & 1 & 0 & 0 & 0 & 1 & $0,00 \%$ \\
\hline & Monokalliapseudes schubarti (Mañe-Garzon, 1949) & 30 & 4 & 27 & 18 & 79 & $0,19 \%$ \\
\hline \multicolumn{8}{|l|}{ SIPUNCULA } \\
\hline SIPUNCULIDEA & Sipunculus sp. & 0 & 0 & 0 & 1 & 1 & $0,00 \%$ \\
\hline \multicolumn{8}{|l|}{ ECHINODERMATA } \\
\hline OPHIUROIDEA & Ophiotrichidae Gen. sp. & 0 & 0 & 0 & 1 & 1 & $0,00 \%$ \\
\hline \multirow[t]{2}{*}{ ECHINOIDEA } & Arbacia lixula (Linnaeus, 1758) & 0 & 1 & 0 & 0 & 1 & $0,00 \%$ \\
\hline & Lytechinus variegatus (Lamarck, 1816) & 0 & 0 & 1 & 3 & 4 & $0,01 \%$ \\
\hline \multirow[t]{2}{*}{ HOLOTUROIDEA } & Holothuria grisea (Selenka, 1867) & 0 & 0 & 2 & 1 & 3 & $0,01 \%$ \\
\hline & Holothuria sp. & 0 & 0 & 0 & 1 & 1 & $0,00 \%$ \\
\hline \multicolumn{8}{|l|}{ ANNELIDA } \\
\hline \multirow[t]{19}{*}{ POLYCHAETA } & Pseudonereis palpata (Treadwell, 1923) & 196 & 459 & 846 & 578 & 2079 & $5,06 \%$ \\
\hline & Nereis sp. & 0 & 1 & 3 & 0 & 4 & $0,01 \%$ \\
\hline & Syllis sp. & 14 & 0 & 1 & 1 & 16 & $0,04 \%$ \\
\hline & Ophisthosyllis sp. & 0 & 0 & 4 & 0 & 4 & $0,01 \%$ \\
\hline & Odontosyllis sp. & 0 & 0 & 1 & 0 & 1 & $0,00 \%$ \\
\hline & Exogone breviantennata Hartmann-Schröder, 1959 & 1 & 2 & 0 & 0 & 3 & $0,01 \%$ \\
\hline & Harmothöe cf. macginitiei Pettibone, 1955 & 1 & 0 & 0 & 5 & 6 & $0,01 \%$ \\
\hline & Halosydna glabra Hartman, 1939 & 1 & 0 & 3 & 17 & 21 & $0,05 \%$ \\
\hline & Podarke sp. & 0 & 0 & 6 & 0 & 6 & $0,01 \%$ \\
\hline & Eulalia sp. & 1 & 0 & 0 & 0 & 1 & $0,00 \%$ \\
\hline & Leodice rubra (Grube, 1856) & 61 & 11 & 32 & 148 & 252 & $0,61 \%$ \\
\hline & Lysidice sp. & 0 & 0 & 0 & 1 & 1 & $0,00 \%$ \\
\hline & Serpulinae Gen sp. & 0 & 0 & 5 & 3 & 8 & $0,02 \%$ \\
\hline & Spirorbinae Gen sp. & 0 & 0 & 0 & 5 & 5 & $0,01 \%$ \\
\hline & Branchiomma sp. & 1 & 0 & 0 & 0 & 1 & $0,00 \%$ \\
\hline & Pherusa sp. & 0 & 0 & 1 & 1 & 2 & $0,00 \%$ \\
\hline & Nicolea uspiana (Nogueira, 2003) & 8 & 10 & 3 & 1 & 22 & $0,05 \%$ \\
\hline & Chaetozone sp. & 1 & 0 & 0 & 0 & 1 & $0,00 \%$ \\
\hline & Scoloplos sp. & 0 & 0 & 1 & 0 & 1 & $0,00 \%$ \\
\hline \multicolumn{8}{|c|}{ PLATYHELMINTHES } \\
\hline RHABDITOPHORA & Stylochoplana divae (Marcus, 1949) & 0 & 1 & 7 & 0 & 8 & $0,02 \%$ \\
\hline \multicolumn{8}{|l|}{ CHORDATA } \\
\hline ACTYNOPTERYGII & Hypleurochilus fissicornis (Quoy \& Gaymard, 1824) & 25 & 1 & 39 & 332 & 397 & $0,97 \%$ \\
\hline TOTAL & & 7979 & 8671 & 19869 & 4563 & 41082 & $100 \%$ \\
\hline
\end{tabular}


Os descritores ecológicos analisados apresentaram diferenças significativas ao longo do período de estudo (Tabela 2). Em novembro foi observada a maior abundância, totalizando 19.869 indivíduos identificados, com média de $2.208 \pm 1.709$ indivíduos presentes em cada corda mexilhoneira amostrada. Em fevereiro foram registradas as maiores contribuições de riqueza (S), índice de diversidade de Shannon $\left(\mathrm{H}^{\prime}\right)$ e equabilidade de Pielou (J'). Neste mesmo período a fauna amostrada apresentou uma baixa dominância (1/D). Por outro lado, os meses de maio e agosto apresentaram as menores riqueza de espécies (S) e índice de diversidade $\left(\mathrm{H}^{\prime}\right)$, e uma maior dominância de espécies (1/D).

Tabela 2 - Média e desvio padrão dos descritores ecológicos da fauna associada às cordas mexilhoneiras na Praia da Cocanha. A mesma letra indica similaridade nos testes estatísticos por período pelo teste SNK ( $p>0.05)$.

\begin{tabular}{lllll}
\hline & Maio & Agosto & Novembro & Fevereiro \\
\hline Abundância (N) & $887^{\mathrm{a}} \pm 597$ & $963^{\mathrm{a}} \pm 858$ & $2.208^{\mathrm{b}} \pm 1709$ & $507^{\mathrm{a}} \pm 485$ \\
Riqueza (S) & $21^{\mathrm{a}} \pm 4$ & $18^{\mathrm{a}} \pm 8$ & $29^{\mathrm{b}} \pm 4$ & $32^{\mathrm{b}} \pm 13$ \\
Diversidade (H') & $2,08^{\mathrm{a}} \pm 0,23$ & $1,89^{\mathrm{a}} \pm 0,55$ & $2,18^{\mathrm{a}} \pm 0,13$ & $2,56^{\mathrm{b}} \pm 0,33$ \\
Dominância (1/D) & $0,17^{\mathrm{a}} \pm 0,03$ & $0,22^{\mathrm{a}} \pm 0,13$ & $0,16^{\mathrm{a}, \mathrm{b}} \pm 0,03$ & $0,12^{\mathrm{b}} \pm 0,04$ \\
Equabilidade (J') & $0,69^{\mathrm{a}} \pm 0,07$ & $0,68^{\mathrm{a}} \pm 0,07$ & $0,65^{\mathrm{a}} \pm 0,04$ & $0,75^{\mathrm{b}} \pm 0,05$ \\
\hline
\end{tabular}

\section{DISCUSSÃO}

\section{Caracterização da fauna vágil associada ao cultivo de mexilhões}

Na praia da Cocanha o semeamento das cordas mexilhoneiras se inicia em janeiro e o período de despesca ocorre principalmente nos meses de dezembro à fevereiro. Exceto para o mês de fevereiro, os resultados indicaram um incremento na abundância da fauna associada conforme o período de imersão das cordas mexilhoneiras na água. Era esperado encontrar um progressivo incremento numérico da fauna associada ao longo do estudo. Entretanto, como a amostragem das cordas foi feita aleatoriamente, em fevereiro foram amostradas algumas cordas repicadas pelos maricultores que provavelmente influenciaram para a redução da abundância neste período. O processo de repicagem é comumente empregado pelos maricultores da Cocanha para a diminuição do fouling e a retirada de organismos plantígrados onde ocorre a limpeza, triagem e confecção de novas cordas mexilhoneiras.

Da mesma forma a riqueza de espécies também aumentou conforme o tempo de permanência das cordas na água, com valores crescentes de maio até fevereiro. Após um período de oito meses de cultivo verificou-se que a riqueza da fauna vágil associada aos mexilhões atingiu valores máximos com uma forte redução na abundância de anfípodas e incremento numérico de grandes predadores, como peixes, gastrópodes e ouriços. Quanto maior o tempo de permanência das cordas mexilhoneiras na água é esperado um aumento da riqueza da fauna associada. O sedimento depositado entre os mexilhões, fragmentos de conchas de organismos mortos e o desenvolvimento do bisso aumentam a heterogeneidade do ambiente, e consequentemente da diversidade de táxons (Tsuchiya \& Nishihira, 1986; Sievers et al., 2014).

Neste estudo foi observada uma notável diversidade e abundância de organismos associados ao cultivo suspenso de mexilhões. O fato das cordas mexilhoneiras estarem suspensas impede o contato da fauna associada com os predadores bentônicos favorecendo o incremento desta comunidade. Em um banco natural de mexilhões a fauna asso- 
ciada representou apenas $7 \%$ da produção total anual da comunidade, composta principalmente por organismos sésseis (Asmus, 1987).

A dominância de anfípodas ocorreu durante todo o período de estudo e foi menos evidente no verão, período onde a comunidade apresentou uma maior equabilidade (Figura 2). A maioria das espécies dominantes de anfípodes presentes neste estudo (Podocerus brasiliensis, Caprella equilibra, Dulichiella anisochir, Jassa marmorata, Monocorophium acherusicum e Ericthonius punctatus) alimenta-se de detritos (GuerraGarcia et al., 2014). Além disso, muitas delas mostram hábito supensívoro, ou alternando o modo de alimentação entre supensívoro e depositívoro dependendo da turbulência da água (Eleftheriou \& Basford, 1989; Dauwe et al., 1998; Dauby et al., 2001). As espécies J. marmorata, M. acherusicum e E. punctatus constroem tubos alimentando-se na entrada dessa estrutura ou gerando correntes de água para dentro do tubo para capturar partículas em suspensão (Beermann, 2014). Podocerus spp. não constroem tubos, porém frequentemente utilizam tubos vazios de outras espécies (Barnard et al., 1988) e alimentam-se de modo similar às espécies de Ericthonius spp. (Dixon \& Moore, 1997). Em ambientes naturais, os bancos de mexilhões promovem uma maior deposição de sedimento fino e matéria orgânica particulada, e em bancos mais antigos observa-se um maior acúmulo de matéria orgânica (Tsuchiya \& Nishihira, 1986). Dessa forma isso poderia explicar a grande abundância desses anfípodes, bem como do aumento da densidade ao longo do tempo.

Os espaços intersticiais criados pelos bancos de mexilhões possuem uma grande variedade de organismos, entretanto são normalmente dominados por poliquetas (Tokeshi, 1995; Cole, 2010), que apresentam uma importante função na estrutura e na dinâmica dessas comunidades. Por exemplo, muitos poliquetas regulam a densidade de outros organismos, como anfípodes, bivalves e outros anelídeos, através da predação, da perturbação do hábitat, e competição (Tita et al., 2000; Tokeshi \& Romero, 2000). A família Nereididae é comumente encontrada em ambientes rasos de regiões estuarinas e do entre-marés (Amaral et al., 2013), muitas espécies ocorrem no costão rochoso e associados a bancos de mexilhões (Hammond \& Griffiths, 2006). Neste estudo a espécie dominante de poliqueta foi o nereidídeo Pseudonereis palpata, já registrada em costões rochosos e bancos de mexilhões do estado de São Paulo (Avellar et al., 2006). Esta espécie provavelmente atue do mesmo modo que P. variegata (Fauchald \& Jumars, 1979), podendo se alimentar de pequenos crustáceos e de macroalgas (Cole et al., 2012).

Os peixes estiveram presentes em todos os períodos de amostragem e foram representados por uma única espécie da família Gobiidae, Hypleurochilus fissicornis. Em fevereiro foi observado um incremento na abundância desta espécie. A ocorrência de $H$. fissicornis e outras seis espécies de peixes também foi verificada em um cultivo comercial de mexilhões no litoral norte de Santa Catarina (Freitas \& Velastin, 2010). 73 espécies de peixes demersais, principalmente da família Gobiidae, foram descritas próximas às fazendas de mexilhões (Iglesias, 1981). Da mesma forma, estudo realizado na Louisiana também relata a presença de gobídeos associados a bancos naturais de ostras (Plunket \& La Peyre, 2005). A estrutura física formada pelas estruturas de cultivo e conchas de bivalves protege os peixes juvenis dos predadores e fornecem substrato para invertebrados sésseis que servem como forrageio para peixes e invertebrados (Dealteris et al., 2004). Em estudo realizado no noroeste da Espanha, os autores verificaram a predominância de crustáceos epibiontes na dieta de gobídeos associados ao cultivo de mexilhões (Lopez-Jamar et al., 1984). 


\section{Implicações ecológicas e suas relações com a produção de mexilhões}

No Atlântico Norte, um estudo identificou cerca de 100 espécies de invertebrados em cordas mexilhoneiras de Mytilus edulis incluindo organismos sésseis (Hickman, 1992). Boa parte desses organismos pode causar a redução do crescimento e da produtividade dos mexilhões através da competição por espaço. Entretanto, medidas para o controle do fouling podem representar consideráveis implicações econômicas para os produtores, atingindo uma média de 14,7\% do custo total anual de operação (Adams et al., 2011).

Com relação aos predadores, alguns dos organismos aqui reportados podem exercer uma importante função no controle biológico do fouling, como os gastrópodes (Minchin \& Duggan, 1989; Roma et al., 2009), ouriços (Ross, 2004; Lodeiros \& Garcia 2004; Roma et al., 2009; Ferreira et al., 2017) e algumas espécies de caranguejos (Hidu et al., 1981; Mallet et al., 2009). Entretanto, determinados peixes, gastrópodes, estrelas do mar e caranguejos fazem dos mexilhões um importante recurso alimentar (Hughes \& Dunkin, 1984; Figueras, 1990; Freire \& González-Gurriaran, 1995; Gosling, 2003; Macreadie et al., 2011), podendo afetar a produtividade das cordas mexilhoneiras. Neste estudo, os caranguejos parecem ser o principal predador dos mexilhões em virtude da grande quantidade de indivíduos observados nos levantamentos. Pequenos mexilhões são especialmente vulneráveis por serem facilmente quebrados por caranguejos de diferentes classes de tamanho, enquanto mexilhões adultos são predados apenas por caranguejos grandes com uma forte quela (Wójcik et al., 2015).

Alguns organismos podem ser aproveitados pelos maricultores, podendo inclusive apresentar algum valor comercial. Peixes, caranguejos, siris, nudibrânquios e poliquetos podem de certa forma, serem aproveitados no mercado de aquariofilia. Outros organismos aqui encontrados, como caranguejos (Hamasaki et al., 2011) e ouriços (Yokota et al., 2002), apresentam um potencial promissor para aquicultura. Além disso, determinados caranguejos, siris, ouriços, e gastrópodes são ocasionalmente consumidos pelos maricultores locais. Organismos sésseis, como por exemplo, ostras perlíferas e ascídias (não avaliados neste estudo) também podem ser utilizados para extração de subprodutos.

Por outro lado, existe a preocupação quanto à dispersão e o estabelecimento de espécies exóticas em áreas de cultivo (Bax et al., 2003). Cordas de mexilhões podem atuar como substratos naturais suscetíveis a fixação de larvas e indivíduos que já se encontram presentes ou estabelecidos nesses ambientes (Macedo et al., 2012). Nesse sentido, as fazendas marinhas fornecem condições propícias para o estabelecimento de novas populações biológicas, inclusive aquelas localizadas próximas a grandes portos de navegação. Neste estudo foi observada a ocorrência do caranguejo exótico Charibdis hellerii em todas as quatro amostragens. Este fato pode estar relacionado à proximidade das fazendas marinhas ao Porto de São Sebastião, provável via de introdução desta espécie (Bernadochi et al., 2012).

Infestações por poliquetas podem reduzir a capacidade dos moluscos de acumular reservas nutricionais (Kent, 1979; Wargo \& Ford 1993), provocar um estresse oxidativo pela abertura frequente das valvas (Chambon et al., 2007) e influenciar na interação predador-presa, deixando os moluscos mais vulneráveis à predação (Kent, 1981; Ambariyanto \& Seed, 1991; Buschbaum et al., 2007).

Os anfípodes representam um importante elo entre os diferentes níveis tróficos da fauna associada às cordas mexilhoneiras. Esses organismos utilizam os espaços e cavidades formados entre os mexilhões como abrigo e refúgio, podendo alimentar-se de detritos, algas ou pequenos crustáceos presentes na superfície dos mexilhões. Ao mesmo 
tempo, peixes, caranguejos e poliquetas aproveitam-se deste importante recurso alimentar, vagando permanentemente pelas cordas ou as utilizando como local de alimentação.

A estrutura da comunidade de organismos vágeis presentes nas cordas mexilhoneiras variou ao longo do tempo, sendo caracterizada pela dominância de anfípodes em todos os períodos analisados. As implicações ecológicas dos organismos vágeis encontrados nas cordas mexilhoneiras e suas relações com a produção comercial de mexilhões aqui apresentadas poderão contribuir para o manejo sustentável da mitilicultura.

Agradecimentos - FAPESP (Proc. 2007/52282-9) e PADI FOUNDATION (RN 150/2007). Agradecemos a colaboração dos pesquisadores: Gustavo Augusto Schmidt de Melo, Marcos Tavares, Luiz Ricardo Lopes de Simone e Carlo Magenta Cunha (MZ-USP); Bruno Garcia (UERJ); Rafael B. de Moura (LABECH/UFRJ); William Onofre e Marcus Rodrigues da Costa (UNIMODULO); Karla Costa e Gabriela Cesquine (UFES); Thiago Cabral Vilar (UFRPE); Artur Ziggiatti Guth (IO-USP); José Luiz Alves (MAPEC). Agradecemos também ao Instituto Costa Brasilis, UNIMÓDULO e MAPEC pelo apoio ao longo do projeto.

\section{REFERÊNCIAS}

Adams, C.M.; Shumway, S.E.; Whitlatch, R.B. \& Getchis, T. Biofouling in Marine Molluscan Shellfish Aquaculture: A Survey Assessing the Business and Economic Implications of Mitigation. Journal of the World Aquaculture Society, Oxford, v. 42, n. 2, p. 242-252, 2011.

Alonso-Pérez, F.; Ysebaert, T. \& Castro, C.G. Effects of suspended mussel culture on benthic-pelagic coupling in a coastal upwelling system (Ría de Vigo, NW Iberian Peninsula). Journal of Experimental Marine Biology and Ecology, Amsterdam, v. 382, n. 2, p. 96-107, 2010.

Amaral, A.C.Z.; Nallin, S.A.H.; Steiner, T.M.; Forroni T.O. \& Filho D.G. Catálogo das espécies de Annelida Polychaeta do Brasil. Editora da UNICAMP, 183 p. Campinas, 2013.

Ambariyanto, A. \& Seed, R. The infestation of Mytilus edulis Linnaeus by Polydora ciliata (Johnston) in the Conwy Estuary, North Wales. Journal of Molluscan Studies, Oxford, v. 57, n. 4, p. 413-424, 1991.

Asmus, H. Secondary production of an intertidal mussel bed community related to its storage and turnover compartments. Marine Ecology Progress Series, Amelinghausen, v. 39, p. 251-266, 1987.

Avellar, L.M.; Nogueira, J.M.M. \& Santos, C.S.G. Biodiversidade de poliquetas nereidídeos (Polychaeta: Nereididae) em costões rochosos do Estado de São Paulo. p. 32, in $1^{\circ}$ Simpósio Latino-Americano de Polychaeta, 2006, São Sebastião, 2006.

Barnard, J.L.; Thomas, J.D. \& Sandved, K.B. Behavior of gammaridean Amphipoda: Corophium, Grandidierella, Podocerus and Gibberosus (American Megaluropus) in Florida. Crustaceana Supplement, Leiden, n. 13, p. 234-244, 1988.

Bax, N.; Williamson, A.; Aguero, M.; Gonzalez, E. \& Geeves, W. Marine invasive alien species: a threat to global biodiversity. Marine Policy, Amsterdan, v. 27, n. 4, p. 313-323, 2003.

Beermann, J. Spatial and seasonal population dynamics of sympatric Jassa species (Crustacea, Amphipoda). Journal of Experimental Marine Biology and Ecology, Amsterdam, v. 459, p. 8-16, 2014. 
Bernadochi L.C.; Silvestri, F. \& Turra, A. Composition and seasonal variation of Brachyura and Anomura (Crustacea, Decapoda) associated with brown mussel farms at Praia da Cocanha. Brazilian Archives of Biology and Technology, Curitiba, v. 55, n. 4, p. 549-557, 2012.

Buschbaum, C.; Buschbaum, G.; Schrey, I. \& Thieltges, D.W. Shell-boring polychaetes affect gastropod shell strength and crab predation. Marine Ecology Progress Series, Amelinghausen, v. 329, p. 123-130, 2007.

Bot-Neto, R.L. \& Rosa, L.C. Ictiofauna associada a um cultivo de ostras no estuário do rio São Francisco, Sergipe, Brasil. Acta of Fisheries and Aquatic Resources, Sergipe, v. 5, p. 59-69, 2017.

Callier, M.D.; Richard, M.; McKindsey, C.W.; Archambault, P. \& Desrosiers, G. Responses of benthic macrofauna and biogeochemical fluxes to various levels of mussel biodeposition: An in situ "benthocosm" experimente. Marine Pollution Bulletin, Amsterdam, v. 58, n. 10, p. 1544-1553, 2009.

Chambon, C.; Legeay, A.; Durrieu, G.; Gonzalez, P.; Ciret, P. \& Massabuau, J.C. Influence of the parasite worm Polydora sp. on the behavior of the oyster Crassostrea gigas: a study of the respiratory impact and associated oxidative stress. Marine Biology, Heidelberg, v. 152, n. 2, p. 329-338, 2007.

Clynick, B.G.; McKindsey, C.W. \& Archambault, P. Distribution and productivity of fish and macroinvertebrates in mussel aquaculture sites in the Magdalen islands (Québec, Canada). Aquaculture, Amsterdam, v. 283, p. 203-210, 2008.

Cole, V.J. Alteration of the configuration of bioengineers affects associated taxa. Marine Ecology Progress Series, Amelinghausen, v. 416, p. 127-136, 2010.

Cole, V.J.; McQuaid, K.A. \& McQuaid, C.D. Examination of small- and large-scale influences on the diet of an omnivorous polychaete indicates weak effects of upwelling. Journal of Experimental Marine Biology and Ecology, Amsterdam, v. 436-437, p. 28-35, 2012.

Comeau, L.A.; Filgueira, R.; Guyondet, T. \& Sonier, R. The impact of invasive tunicates on the demand for phytoplankton in longline mussel farms. Aquaculture, Amsterdam, v. 441, p. 95-105, 2015.

Dauby, P.; Scailteur, Y. \& De Broyer, C. Trophic diversity within the eastern Weddell Sea amphipod community. Hydrobiologia, Brussels, v. 443, n. 1, p. 69-86, 2001.

Dauwe, B.; Herman, P.M.J. \& Heip, C.H.R. Community structure and bioturbation potential of macrofauna at four North Sea stations with contrasting food supply. Marine Ecology Progress Series, Amelinghausen, v. 173, p. 67-83, 1998.

Dealteris, J.T.; Kilpatrick, B.D. \& Rheault, R.B. A comparative evaluation of the habitat value of shellfish aquaculture gear, submerged aquatic vegetation and a non-vegetated seabed. Journal of Shellfish Research, Washigton, v. 23, p. 867-874, 2004.

Dixon, I.M.T. \& Moore, P.G. A comparative study on the tubes and feeding behaviour of eight species of corophioid Amphipoda and their bearing on phylogenetic relationships within the Corophioidea. Philosophical Transactions of the Royal Society of London. Series B, London, v. 352, n. 1349, p. 93-112, 1997.

Eleftheriou, A. \& Basford, D.J. The macrobenthic infauna of the offshore Northern North Sea. Journal of the Marine Biological Association of the United Kingdom, Cambridge, v. 69, n. 1, p. 123-143, 1989. 
Fauchald, K. \& Jumars, P.A. The diet of worms: a study of polychaete feeding guilds. Oceanography and Marine Biology. An anual review, Aberdeen, v. 17, p. 193-284, 1979.

Ferreira, V.M.; Pereira, M.B.; Amorim, M.A.S.; Cruz, G.S. \& Borges, C.L.L. Uso de controle biológico para a fauna acompanhante no cultivo da vieira Nodipecten nodosus (Linnaeus, 1758). Semioses, Rio de Janeiro, v. 11, p. 08-16, 2017.

Figueras, A. Mussel culture in Spain. Marine Behaviour and Physiology, London, v. 16, n. 3, p. 177-209, 1990.

Fitridge, I.; Dempste, T.; Guenther, J. \& de Nys, R. The impact and control of biofouling in marine aquaculture: a review. Biofouling, London, v. 28, n. 7, p. 649-669, 2012.

Freire, J. \& González-Gurriaran, E. Feeding ecology of the velvet swimming crab Necora puber in mussel raft areas of the Ria de Arousa (Galicia, NW Spain). Marine Ecology Progress Series, Amelinghausen, v. 119, p. 139-154, 1995.

Freitas, M.O. \& Velastin, R. Ictiofauna associada a um cultivo de mexilhão Perna perna (Linnaeus, 1758) Norte Catarinense, Sul do Brasil. Acta Scientiarum Biological Sciences, Maringá, v. 32, n. 1, p. 31-37, 2010.

Gibbs, M.T. Interactions between bivalve shellfish farms and fishery resources. Aquaculture, Amsterdam, v. 240, p. 267-296, 2004.

Gosling, E. Ecology of Bivalves, p. 44-86, in Gosling, E. (ed.), Bivalve Molluscs - Biology, Ecology and Culture. Blackwell Publishing, 443 p., New Jersey, 2003.

Guerra-Garcia, J.M.; Figueroa, J.M.T.; Navarro-Barranco, C.; Ros, M.; Sánchez-Moyano, J.E. \& Moreira, J. Dietary analysis of the marine Amphipoda (Crustacea: Peracarida) from the Iberian Peninsula. Journal of Sea Research, Amsterdam, v. 85, p. 508-517, 2014.

Hamasaki, K.; Obata, Y.; Dan, S. \& Kitada, S. A review of seed production and stock enhancement for commercially important portunid crabs in Japan. Aquaculture International, Heidelberg, v. 19, n. 2, p. 217-235, 2011.

Hammond, W. \& Griffiths, C.L. Biogeographical patterns in the fauna associated with southern African mussel bed. African Zoology, Oxford, v. 41, n. 1, p. 123-130, 2006.

Hickman, R.W. Mussel Cultivation, p. 425-510, in Gosling, E. (ed.), The Mussel Mytilus: ecology, physiology, genetics and culture. Elsevier, 589 p., Amsterdam, 1992.

Hidu, H.; Conary, C. \& Chapman, S.R. Suspend culture of oysters: biological fouling control. Aquaculture, Amsterdam, v. 22, p. 189-192, 1981.

Hughes, R.N. \& Dunkin, S.B. Behavioural components of prey selection by dogwhelks, Nucella lapillus (L.), feeding on mussels, Mytilus edulis L., in the laboratory. Journal of Experimental Marine Biology and Ecology, Amsterdam, v. 77, p. 45-68, 1984.

Iglesias, J. Spatial and temporal changes in the demersal fish community of the Ria de Arosa (NW Spain). Marine Biology, Berlim, v. 65, n. 2, p. 199-208, 1981.

Kamermans, P.; Blankendaal, M. \& Perdon, J. Predation of shore crabs (Carcinus maenas (L.)) and starfish (Asterias rubens L.) on blue mussel (Mytilus edulis L.) seed from wild sources and spat collectors. Aquaculture, Amsterdam, v. 290, n. 3-4, p. 256-262, 2009.

Kent, R.M.L. The influence of heavy infestations of Polydora ciliata on the flesh content of Mytilus edulis. Journal of the Marine Biological Association of the United Kingdom, Cambridge, v. 59, n. 2, p. 289-297, 1979. 
Kent, R.M.L. The effect of Polydora ciliata on the shell strength of Mytilus edulis. ICES Journal of Marine Sciences, Oxford, v. 39, n. 3, p. 252-255, 1981.

Lacoste, E.; Le Moullac, G.; Levy, P.; Gueguen, Y. \& Gaertner-Mazouni, N. Biofouling development and its effect on growth and reproduction of the farmed pearl oyster Pinctada margaritifera. Aquaculture, Amsterdam, v. 434, p. 18-26, 2014.

Lodeiros, C. \& Garcia, N. The use of sea urchins to control fouling during suspended culture of bivalves. Aquaculture, Amsterdam, v. 231, p. 293-298, 2004.

Lopez-Jamar, E.; Iglesias, J. \& Otero, J.J. Contribution of infauna and mussel-raft epifauna to demersal fish diet. Marine Ecology Progress Series, Amelinghausen, v. 15, p. 13-18, 1984.

Macedo, P.P.B.; Masunari, S. \& Corbetta, R. Decapod crustaceans associated with farming ropes of the mussel Perna perna (Linnaeus, 1758) (Mollusca, Bivalvia, Mytilidae) from Armação do Itapocoroy Bay, Penha - SC. Biota Neotropica, Campinas, v. 12, n. 2, p. 185-195, 2012.

Macreadie, P.I.; Geraldi, N.R. \& Peterson, C.H. How small-scale variation in oyster reef patchiness influences predation on bivalves. Marine Ecology Progress Series, Amelinghausen, v. 429, p. 87-91, 2011.

Mallet, J.F.; LeBlanc, A.R.; Ouellette, M. \& Comeau, L.A. Abundance and function of rock crabs (Cancer irroratus) in longline mussel (Mytilus edulis) farms. Department of Fisheries and Oceans Canada, 53 p. New Brunswick, 2009.

Marenzi, A.W.C. \& Branco, J.O. O cultivo do mexilhão Perna perna no município de Penha, SC, p. 227-244, in Branco, J.O. \& Marenzi, A.W.C. (eds.), Bases ecológicas para um desenvolvimento sustentável: estudos de caso em Penha, SC. Editora da Univali, 292 p., Itajaí, 2006.

Marques, H.L.A. Criação comercial de mexilhões. Nobel, 111 p., São Paulo, 1998.

McKindsey, C.W.; Lecuona, M.; Huot, M. \& Weise, A.M. Biodeposit production and benthic loading by farmed mussels and associated tunicate epifauna in Prince Edward Island. Aquaculture, Amsterdam, v. 295, p. 44-51, 2009.

Metri, R., Rocha, R.M. \& Marenzi, A. Epibiosis reduction on productivity in a mussel culture of Perna perna (Linné, 1758). Brazilian Archives of Biology and Technology, Curitiba, v. 45, n. 3, p. 325-31, 2002.

Minchin, D. \& Duggan, C.B. Biological Control of the Mussel in Shellfish Culture. Aquaculture, Amsterdam, v. 81, p. 97-100, 1989.

Morrissey, J.; Cole, R.G.; Davey, N.K.; Handley, S.J.; Bradley, A.; Brown, S.N. \& Madarasz, A.L. Abundance and diversity of fish on mussel farms in New Zealand. Aquaculture, Amsterdam, v. 252, p. 277-288, 2006.

Peteiro, L.G.; Filgueira, R.; Labarta, U. \& Fernández-Reiriz, J.F. The role of fish predation on recruitment of Mytilus galloprovincialis on different artificial mussel collectors. Aquacultural Engineering, Amsterdam, v. 42, n. 1, p. 25-30, 2010.

Plunket, J. \& La Peyre, M.K. Oyster beds as fish and macroinvertebrate habitat in Barataria bay, Louisiana. Bulletin of Marine Science, Miami, v. 77, n. 1, p. 155-164, 2005.

Rajbanshi, R. \& Pederson, J. Competition among invading ascidians and a native mussel. Journal of Experimental Marine Biology and Ecology, Amsterdam, v. 342, n. 1, p. 163-165, 2007. 
Rocha, R.M.; Kremer, L.P.; Baptista, M.S. \& Metri, R. Bivalve cultures provide habitat for exotic tunicates in southern Brazil. Aquatic Invasions, Helsinki, v. 4, n. 1, p. 195-205, 2009.

Roma, R.P.C.R.; Marques, H.L.A. \& Bueno, R.S. Controle biológico de organismos incrustantes em um cultivo de vieiras Nodipecten nodosus (Linnaeus, 1758) em Ubatuba, SP, Brasil. Biotemas, Florianópolis, v. 22, n. 4, p. 107-115, 2009.

Ross, K.A.; Thorpe, J.P. \& Brand, A.R. Biological control of fouling in suspended scallop cultivation. Aquaculture, Amsterdam, v. 229, p. 99-116, 2004.

Sá, F.S.; Nalesso, R.C. \& Paresque, K. Fouling organisms on Perna perna mussels: is it worth removing them? Brazilian Journal of Oceanography, São Paulo, v. 55, n. 2, p. 155-161, 2007.

Sievers, M.; Dempster, T.; Fitridge, I. \& Keough, M. J. Monitoring biofouling communities could reduce impacts to mussel aquaculture by allowing synchronisation of husbandry techniques with peaks in settlement. Biofouling, London, v. 30, n. 2, p. 203-212, 2014.

Silvestri, F.; Bordon, I.C.A.C.; Bueno, R.S.; Turra, A. \& Marques, H.L.A. Cultivo de bivalvos en el estado de San Pablo, Brasil. Infopesca Internacional, Montevidéu, v. 35, p. 36-39, 2008.

Silvestri, F. \& Turra, A. Excrement production by brown mussels Perna perna (L. 1758) under static conditions. Pan-American Journal of Aquatic Sciences, Montevideo, v. 8, n. 1, p. 30-38, 2013.

Souza-Conceição, J.M.; Castro-Silva, M.A.; Huergo, G.P.; Soares, G.S.; Marenzi, A.C. \& Manzoni, G.C. Associação da ictiofauna capturada através de rede de emalhe com o cultivo de mexilhões da enseada de Armação do Itapocoroy, em Penha (Santa Catarina - Brasil). Boletim do Instituto de Pesca, São Paulo, v. 29, n. 2, p. 117-121, 2003.

Tallman, J.C. \& Forrester, G.E. Oyster grow-out cages function as artificial reefs for temperate fishes. Transactions of the American Fisheries Society, Oxford, v. 136, n. 3, p. 790-799, 2007.

Tita, G.; Desrosiers, G.; Vincx, M. \& Nozais, C. Predation and sediment disturbance effects of the intertidal polychaete Nereis virens (Sars) on associated meiofaunal assemblages. Journal of Experimental Marine Biology and Ecology, Amsterdam, v. 243, n. 2, 261-282, 2000.

Tokeshi, M. Polychaete abundance and dispersion patterns in mussel beds: a non-trivial 'infaunal' assemblages on a Pacific American rocky shore. Marine Ecology Progress Series, Amelinghausen, v. 125, p. 137-147, 1995.

Tokeshi, M. \& Romero, L. Spatial overlap and coexistence in a mussel-associated polychaete assemblage on a South American rocky shore. Marine Ecology, New Jersey, v. 21, n. 3-4, p. 247-261, 2000.

Tsuchiya, M. \& Nishihira, M. Islands Mytilus edulis as a habitat for small intertidal animals: effect of Mytilus age structure on the species composition of the associated fauna and community organization. Marine Ecology Progress Series, Amelinghausen, v. 31, p. 171-178, 1986.

Underwood, A.J. Experiments in Ecology: their logical design and interpretation using analyses of variance. Cambridge University Press, 522 p., Cambridge, 1997.

Wargo, R.N. \& Ford, S.E. The effect of shell infestation by Polydora sp. and infection by Haplosporidium nelsoni (MSX) on the tissue condition oysters Crassostrea gigas. Estuaries, Chesapeake, v. 16, p. 229-234, 1993. 
Watson, D.I.; Shumway, S.E. \& Whitlatch, R.B. Biofouling and the shellfish industry, p. 317-337, in Shumway S.E. \& Rodrick G.E. (eds.) Shellfish Safety and Quality. Woodhead Publishing, 612 p., Cambrigde, 2009.

Witman, J.D. \& Suchanek, T.H. Mussels in flow: drag and dislodgement by epizoans. Marine Ecology Progress Series, Amelinghausen, v. 16, p. 259-268, 1984.

Wójcik, D.; Normant, M.; Dmochowska, B. \& Fowler, A. Impact of Chinese mitten crab Eriocheir sinensis on blue mussel Mytilus edulis trossulus - laboratory studies of claw strength, handling behavior, consumption rate, and size selective predation. Oceanologia, Warszawa, v. 57, n. 3, p. 263-270, 2015.

Yokota, Y.; Matranga, V. \& Smolenicka, Z. The Sea Urchin: From Basic Biology to Aquaculture. Balkema, 239 p., Lisse, 2002. 